\title{
Spontaneous giant right coronary artery pseudoaneurysm
}

\author{
Yi-Chia Wang, MD, ${ }^{\mathrm{a}}$ Ron-Bin Hsu, MD, ${ }^{\mathrm{b}}$ and Chi-Hsiang Huang, $\mathrm{MD}^{\mathrm{a}}$
}

A healthy 35-year-old woman who denied any trauma or surgical history reported progressive dyspnea for 1 month. Because of exercise intolerance, she was intubated. Her symptoms rapidly improved in 1 day without any intervention. Her chest radiograph showed cardiomegaly, and chest computed tomography revealed a large, lobulated right heart tumor with rich contrast enhancement (Figure 1, A). Thoracoscopic pericardiotomy for biopsy showed marked fibrosis, lymphocyte infiltration, and no evidence of malignancy. The patient's condition was stable, and no exercise intolerance was noted in the subsequent 3 months. Follow-up chest computed tomography showed cardiac mass size reduction, and a connection from the right coronary artery (RCA) to the cardiac mass was noted (Figure 1, B). Coronary angiography showed total RCA occlusion. The RCA proximal orifice was noted, but the distal

From the Department of Anesthesiology, ${ }^{\mathrm{a}}$ National Taiwan University Hospital, Taipei, Taiwan; and the Department of Surgery, ${ }^{\mathrm{b}}$ National Taiwan University Hospital, Taipei, Taiwan.

Disclosures: Authors have nothing to disclose with regard to commercial support. Received for publication Dec 8, 2013; accepted for publication Dec 17, 2013; available ahead of print Jan 31, 2014.

Address for reprints: Chi-Hsiang Huang, MD, Department of Anesthesiology, National Taiwan University College of Medicine and National Taiwan University Hospital, 7 Chung-Shan South Rd, Taipei, Taiwan, 10002 (E-mail: tee.ntuh@gmail.com).

J Thorac Cardiovasc Surg 2014;148:349-50

$0022-5223 / \$ 36.00$

Copyright (C) 2014 by The American Association for Thoracic Surgery http://dx.doi.org/10.1016/j.jtcvs.2013.12.023 end was not found. Perioperative transesophageal echocardiography demonstrated RCA pseudoaneurysm with shunting from the proximal RCA orifice (Figure 2, $A$ and $B$ ) and pseudoaneurysm rupture into the right ventricle at the level of tricuspid valve (Figure 2, C). During the operation, a single huge proximal RCA pseudoaneurysm $(14 \times 10 \mathrm{~cm})$ was found. (Figure 3, A). There was organized thrombus formation within the pseudoaneurysm. The patient's tricuspid valve function was preserved after RCA pseudoaneurysm excision and Hemashield (Maquet Holding BV \& Co KG, Rastatt, Germany) patch repair for RV rupture (Figure 3, $B)$. Coronary artery bypass grafting with great saphenous vein to the posterior descending artery was performed. The patient was extubated the same day after surgery and had smooth recovery.

Giant coronary artery pseudoaneurysm is a rare event; usually caused by catheter-based intervention, surgical complication, blunt trauma, or infection. Rapidly enlarging pseudoaneurysm carries the risk of rupture and tamponade. Thrombosis and subsequent distal embolization is also a problem. ${ }^{1}$ Patients have variable presentations, ranging from asymptomatic to myocardial infarction, ventricular arrhythmia, or respiratory symptoms. There is no wellestablished guideline for surgical treatment of spontaneous coronary pseudoaneurysm because of the rare incidence and poorly understood disease course. ${ }^{2}$ Treatment should be individualized. Our patient underwent successful surgical repair and coronary revascularization.

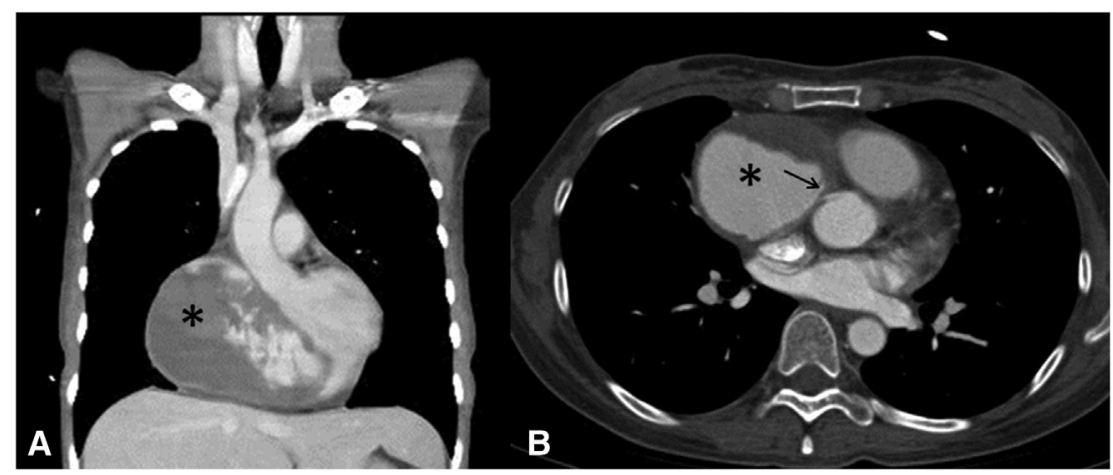

FIGURE 1. A, Computed tomography shows a huge, right-sided cardiac mass (asterisk) with contrast enhancement and thrombus formation. B, The pseudoaneurysm (asterisk) originates from the right coronary artery (arrow), as is clearly visible. 


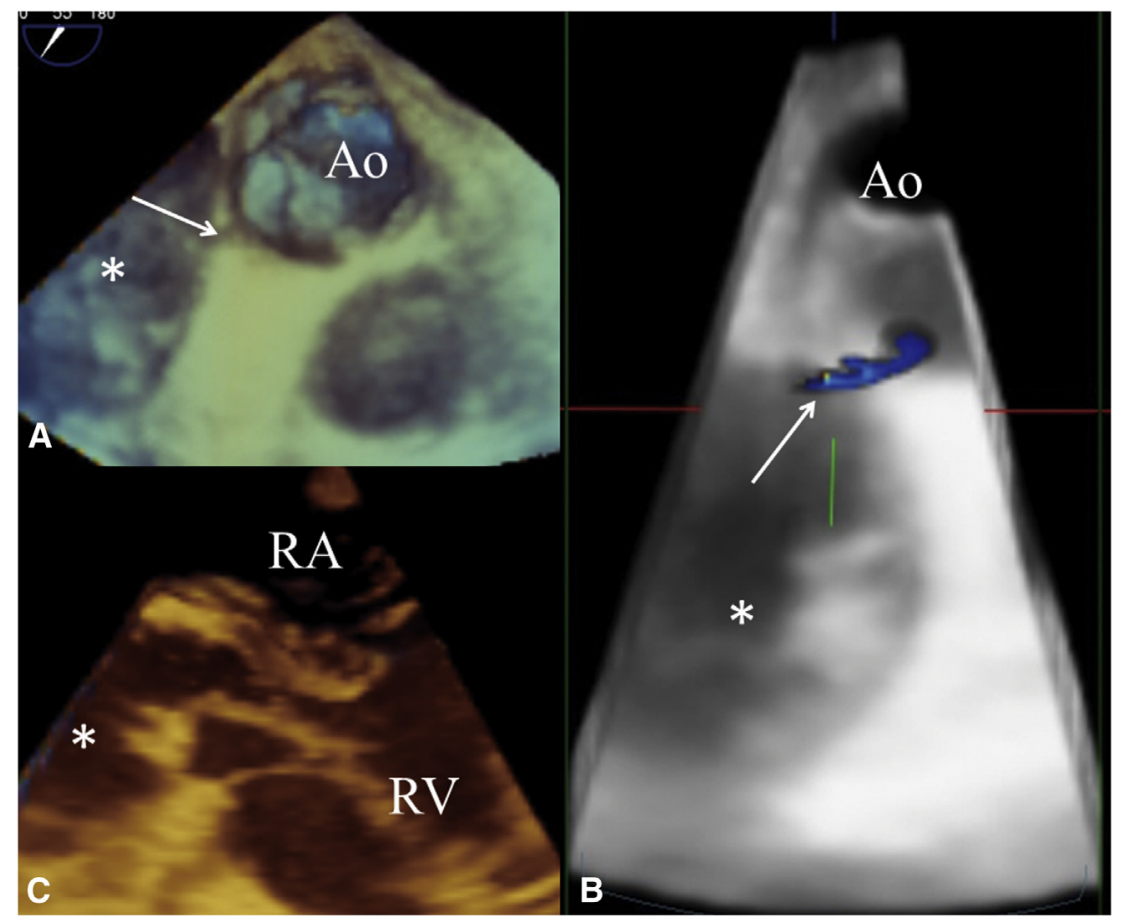

FIGURE 2. A, Three-dimensional transesophageal echocardiography shows the pseudoaneurysm (asterisk) to come from the proximal right coronary artery orifice (arrow). B, Color 3-dimensional transesophageal echocardiography reveals the shunting from the right coronary artery (arrow). C, Pseudoaneurysm (asterisk) ruptured into the right ventricle $(R V)$ at the tricuspid valve level. Ao, Aorta; $R A$, right atrium.

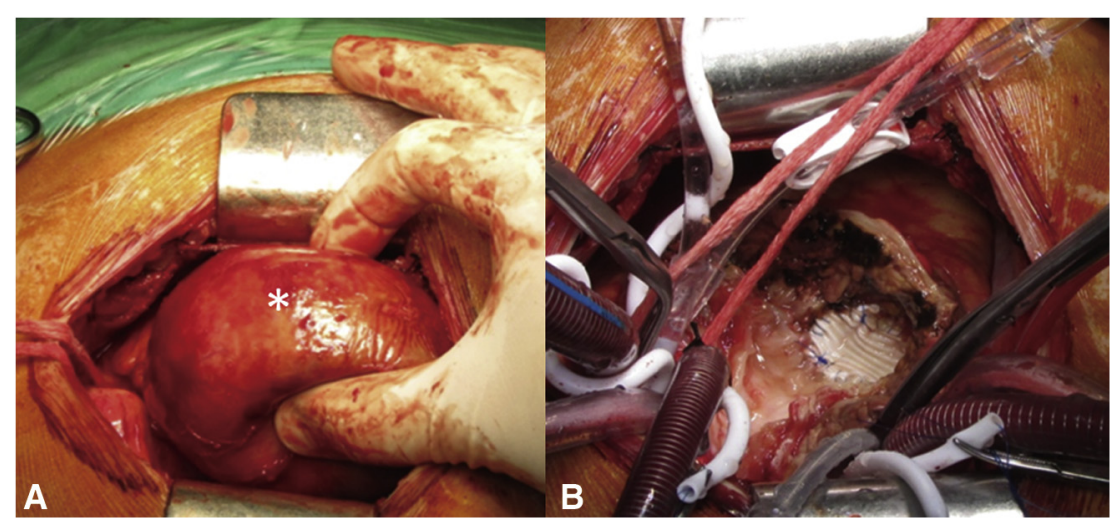

FIGURE 3. A, Operative photograph shows a huge proximal right coronary artery pseudoaneurysm (asterisk) $14 \times 10 \mathrm{~cm}$ in size. B, Operative photograph demonstrates surgical excision of the right coronary artery pseudoaneurysm and Hemashield patch repair for right ventricular rupture.

\section{References}

1. Izutani H, Shibukawa T, Kawamoto J, Ishibashi K. Spontaneous right coronary artery pseudoaneurysm. Gen Thorac Cardiovasc Surg. 2007;55:259-61.

2. Aqel RA, Zoghbi GJ, Iskandrian A. Spontaneous coronary artery dissection, aneurysms, and pseudoaneurysms: a review. Echocardiography. 2004;21: 175-82. 